\title{
Modélisation élastoplastique de la densification des verres de silice sous des sollicitations de contact à l'échelle micrométrique
}

\author{
Guillaume Kermouche ${ }^{1, a}$, Étienne Barthel ${ }^{2}$, LaËtitia Tang ${ }^{3}$ \\ ET DAMIEN VANDEMBRoucQ ${ }^{2}$ \\ 1 Laboratoire de Tribologie et Dynamique des Systèmes, UMR 5513 CNRS/ECL/ENISE/ENSMSE, \\ École Nationale d'Ingénieurs de Saint-Étienne, 58 rue Jean Parot, 42023 Saint-Étienne Cedex 2, France \\ 2 Surface du verre et interfaces, UMR 125 CNRS/Saint-Gobain, 39 quai Lucien Lefranc, 93303 Aubervilliers Cedex, France \\ 3 ESI Group, ESI France - ESI Group, Le Récamier, 70 rue Robert, 69458 Lyon Cedex 06, France
}

Reçu le 25 mai 2007, accepté le 24 janvier 2008

\begin{abstract}
Résumé - Les verres de silice rompent de manière fragile aux échelles supérieures au micron mais présentent une réponse plastique aux échelles inférieures. Plus particulièrement, ils se densifient de façon irréversible sous des sollicitations de contact. Dans cet article, nous présentons une loi de comportement mécanique permettant de reproduire ce phénomène. Les paramètres matériaux sont identifiés par comparaison entre des résultats de simulations numériques par éléments-finis de l'essai de nanoindentation et des résultats expérimentaux telles que la courbe force-enfoncement et une cartographie de densification obtenue par spectroscopie Raman. Nous utilisons ensuite cette loi pour prédire la distribution de densification obtenue après indentation Vickers tridimensionnelle et indentation Cube Corner. Une simulation numérique de rayure est aussi effectuée permettant d'obtenir une première idée de la densification de ces matériaux sous ce type de sollicitations. Ces différents résultats montrent que la zone densifiée sous ces différents types de chargement est toujours de l'ordre de la taille du contact.
\end{abstract}

Mots clés : Verre / matériaux poreux / nanoindentation / rayure / densification / éléments-finis

\begin{abstract}
Computational modeling of the densification of silicate glasses under contact loadings at the micron scale. Silicate glasses are known to be brittle at the macroscopic scale but they can also undergo plastic deformation at the microscopic scale. More specifically, plastic densification occurs under contact loadings. In this paper, a constitutive model, which allows to take into account this phenomenon, is presented. An identification of the material parameters using instrumented indentation results is proposed. This identification is based on the comparison of local residual indentation-induced densification field obtained using Raman spectroscopy and computational results. This constitutive model is then used to analyse the distribution of densification after three-dimensional Vickers indentation and Cube Corner indentation. Finally, a finite-element analysis of the scratch test is done to show the plastic densification of such materials under scratching conditions. These different results show that the size of the densified zone under indentation-scratch loading is strongly related to the contact size.
\end{abstract}

Key words: Glass / pressure dependent plasticity / nanoindentation / scratch / densification / finite-element method

\section{Introduction}

La silice et les verres silicatés sont des archétypes de matériaux fragiles [1]. Mais si leur fragilité s'entend à l'échelle macroscopique, il existe une taille caractéristique, de l'ordre de quelques microns, en dessous de laquelle la

a Auteur pour correspondance :

guillaume.kermouche@enise.fr déformation plastique est plus favorable que la propagation de fissure [2-5]. Aux échelles plus faibles, ce comportement en déformation irréversible des matériaux vitreux est en outre caractérisé par un phénomène plus ou moins important de densification, en plus de l'écoulement en cisaillement classiquement observé dans les matériaux métalliques $[5,6]$. D'un point de vue physique, le verre de silice est composé de tétraèdres $\mathrm{SiO}_{4}$ (ordre à courte 
distance) qui sont organisés sous forme de boucle fermée (anneaux). Cette organisation traduit l'existence d'un volume libre plus ou moins important en fonction du nombre de tétraèdres par anneaux. C'est finalement ce volume libre qui pourrait expliquer la possibilité que possède ce matériau à densifier de manière irréversible [7-10].

Bien que connu depuis un demi-siècle, ce dernier phénomène reste mal compris; maîtrisé, il pourrait ouvrir des perspectives technologiques nouvelles tant pour l'amélioration de la réponse mécanique des verres que pour leur mise en forme à l'échelle locale. Par exemple, la compréhension et la maîtrise du poids relatif de la densification et de l'écoulement en cisaillement permettraient de mieux contrôler la rayabilité de la surface des verres en limitant l'apparition de fractures sous l'effet de la rayure.

Le peu d'étude sur ce sujet s'explique par la nécessité d'utiliser des mesures mécaniques à l'échelle micronique. Avec les récents développements des techniques de nanoindentation instrumentée [11-13], cet objectif semble devenir réalisable. Cependant la simple analyse des résultats d'indentation instrumentée (force-pénétration) ne permet pas à elle seule de statuer sur le comportement mécanique des matériaux indentés car il s'agit de quantités intégrales. Récemment, des résultats expérimentaux infiniment plus riches ont été apportés : des cartes de distribution de densification de la silice vitreuse après microindentation ont été obtenues à l'aide de techniques de microspectroscopie Raman [7,14]. Ces travaux ont en outre permis de caractériser le durcissement de ces matériaux sous compression hydrostatique et ainsi de préciser la loi d'écrouissage.

Un ensemble riche et complexe de données est aujourd'hui à notre disposition et l'utilisation de la méthode des éléments-finis semble être un des moyens d'investigation les plus intéressants pour les analyser. L'objectif est, à terme, de proposer une loi de comportement pour décrire la déformation plastique des verres silicatés à l'échelle micronique et ainsi de pouvoir mieux appréhender les relations entre zone densifiée sous contact et résistance mécanique du verre.

\section{Mesure de densification par spectroscopie Raman}

Le spectre Raman de la silice présente deux «pics » dont la position et l'intensité varient de façon détectable lorsque le matériau est densifié (Fig. 1). Il semble maintenant possible de relier ce décalage $[15,16]$ à la variation relative de densité à partir de la relation suivante :

$$
0,143 \log _{10}\left(\frac{\rho}{\rho_{0}}\right)=\log _{10}\left(\frac{v}{v_{0}}\right)
$$

avec $\rho$ et $\rho_{0}$ les densités actuelles et initiales, $v$ et $v_{0}$ étant les positions actuelles et initiales du pic $D_{2}$. Cette technique permet donc d'établir des distributions de densité de la silice avec une résolution spatiale d'environ $2 \mu \mathrm{m}$. Perriot et al. [14] ont analysé des indents d'environ $30 \mu \mathrm{m}$ de côté, afin de respecter un compromis entre résolution

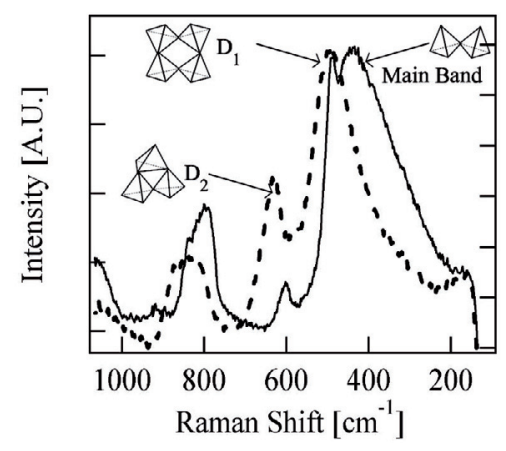

Fig. 1. Spectres Raman [2,7] de silice fondue (trait plein) et de silice fondue puis densifiée d'environ $20 \%$ par indentation (pointillés).

spatiale de la méthode de détection et fissuration excessive des indents. En effet, pour cette taille d'indent, une fissuration limitée apparaît, et par conséquent, il est possible de faire l'hypothèse qu'elle n'altère pas sensiblement le phénomène de densification. La densification peut alors être définie comme la trace du tenseur des petites déformations :

$$
1-\frac{\rho}{\rho_{0}}=\operatorname{tr}(\overline{\bar{\varepsilon}})=3 \varepsilon_{\mathrm{m}}
$$

\section{2 Élaboration d'une loi de comportement adaptée}

\subsection{Données expérimentales}

Les mesures de densité et les courbes de chargedécharge obtenues par indentation instrumentée sont des premiers outils pour déterminer une loi de comportement pour ces verres. Cependant, ces résultats ne sont pas encore suffisants étant donné le peu de connaissance relative à l'écrouissage de la silice fondue, tant au point de vue déviatorique que sphérique. Dans ce but, Perriot [7] a caractérisé le durcissement de ces matériaux sous compression hydrostatique à l'aide d'un dispositif à enclume diamant [17] et de mesures de densification par spectroscopie Raman. Il a ainsi précisé la loi d'écrouissage du point de vue purement hydrostatique. Dans une première approche, nous avons considéré un écrouissage linéaire permettant de représenter les premiers stades de déformations jusqu'à la saturation de la densification [18].

\subsection{Hypothèses du modèle}

Les phénomènes de densification sont couramment rencontrés dans les problèmes mettant en jeu des matériaux poreux. Dans ce cadre, de nombreuses lois de comportement ont été développées. Citons, par exemple, les lois de type Cam-Clay pour modéliser le comportement de certains sols et roches [19] ou encore les critères 


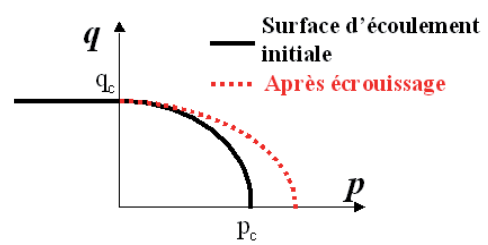

Fig. 2. Illustration du critère de plasticité adopté.

d'écoulement de type Gurson pour modéliser la compaction à chaud de poudres métalliques [20].

Pour modéliser la densification des verres de silice à l'échelle micronique, nous avons choisi un critère de plasticité proche de celui de Shima-Oyane [21] couplé à une loi d'écoulement associée.

$$
q^{2}+\left(q_{\mathrm{c}} / p_{\mathrm{c}}\right)^{2} p^{2}-q_{\mathrm{c}}^{2}=0 \text { pour } p \geq 0
$$

avec $q$ la contrainte équivalente de von Mises, $p$ la pression hydrostatique, $q_{\mathrm{c}}$ et $p_{\mathrm{c}}$ des paramètres matériaux représentant les limites d'élasticité en cisaillement et pression. Dans le cas où la pression hydrostatique est négative (traction), le critère précédent est réduit à celui de von Mises (Fig. 2). Le durcissement hydrostatique est caractérisé par une évolution $p_{\mathrm{c}}$ avec la densification irréversible. Dans une première approche, nous pouvons supposer que la densification se sépare additivement en une part réversible (élastique) et une part irréversible (plastique). Une mesure de la densification irréversible est alors la trace du tenseur des déformations plastiques. Pour décrire ce durcissement hydrostatique, nous avons choisi une évolution linéaire de $p_{\mathrm{c}}$ en fonction de la déformation plastique moyenne $\varepsilon_{\mathrm{m}}^{\mathrm{p}}=(1 / 3) \operatorname{tr}\left(\varepsilon^{\mathrm{p}}\right)$, au regard des résultats de Perriot et al. [7-14].

$$
p_{\mathrm{c}}=\alpha \varepsilon_{\mathrm{m}}^{\mathrm{p}}+p_{\mathrm{c} 0}
$$

avec $p_{\mathrm{c} 0}=11,5 \mathrm{GPa}$ et $\alpha=-100 \mathrm{GPa}$ des constantes matériaux identifiées à partir des résultats de Perriot [7]. Remarquons, à ce stade, que le module d'écrouissage volumique $\alpha$ est de l'ordre de grandeur du module d'élasticité volumique : $K=120 \mathrm{GPa}$. Ce résultat peut paraître surprenant lorsque l'on prend comme référence les matériaux métalliques qui ont des modules d'écrouissage de l'ordre du centième du module d'élasticité. Cependant, il faut bien garder à l'esprit que la plasticité du verre de silice ne repose pas sur un mécanisme de type dislocations, par conséquent une telle analogie n'a pas de sens. Les résultats de Perriot [7] montrent que le module d'écrouissage augmente avec la densification. À partir d'une certaine valeur de densification, le module d'écrouissage devient très proche du module d'élasticité. Il est alors plus facile de déformer élastiquement le matériau que plastiquement, ce qui se traduit alors par une saturation de la densification du matériau. Selon Perriot [7], Perriot et al. [14], ce phénomène de saturation apparaît à partir d'une valeur de densification de l'ordre de $20 \%$.

\subsection{Implémentation}

Cette loi de comportement a été implémentée dans le code par éléments-finis Systus ${ }^{\circledR}[22]$ en utilisant une formulation lagrangienne réactualisée [23]. Le problème à résoudre pour implémenter une loi de comportement élastoplastique de façon implicite est le suivant :

Pour un point d'intégration donné, connaissant le tenseur des déformations aux instant $t$ et $t+\Delta t$ ainsi que le tenseur des contraintes à l'instant $t$, déterminer le tenseur des contraintes à l'instant $t+\Delta t$ telle que la loi de comportement soit rigoureusement satisfaite à l'instant $t+\Delta t$.

Pour cela, nous utilisons un algorithme de retour classique [23]. À partir de la condition de consistance et de la loi d'écrouissage, nous obtenons un système d'équations non-linéaires :

$$
\begin{aligned}
& f_{1}\left(\Delta \lambda, \Delta \varepsilon_{\mathrm{m}}^{\mathrm{p}}\right)=0 \\
& f_{2}\left(\Delta \lambda, \Delta \varepsilon_{\mathrm{m}}^{\mathrm{p}}\right)=0
\end{aligned}
$$

avec $\Delta \varepsilon_{\mathrm{m}}^{\mathrm{p}}$ la variation de déformation plastique moyenne et $\Delta \lambda$ la variation du multiplicateur plastique entre les instants $t$ et $t+\Delta t$. Ce système est résolu à l'aide d'une méthode de type Newton-Raphson.

\section{Simulation numérique de l'essai d'indentation}

Le durcissement hydrostatique ayant été mesuré expérimentalement, le seul paramètre inconnu est la valeur de la limite d'élasticité en cisaillement $q_{\mathrm{c}}$. D'un point de vue expérimental, nous disposons des courbes de charge-décharge de nanoindentation instrumentée effectuées sur des échantillons de silice fondue avec des pointes de type Berkovich (les pointes Vickers étant utilisées en micro-indentation) ainsi que des cartographies de densification obtenues par spectroscopie Raman après indentation Vickers. Les différentes géométries d'indenteurs utilisées couramment en indentation sont décrites en figure 3. Nous avons choisi de déterminer ce paramètre par une analyse inverse reposant sur la comparaison des courbes charge-décharge obtenues numériquement et expérimentalement avec une pointe Berkovich (Fig. 4). Avec cette méthode, nous obtenons une valeur 6,5 GPa pour la limite d'élasticité en cisaillement du matériau considéré. Des compléments sur la démarche adoptée pour identifier ce paramètre peuvent être trouvés dans [18]. Notons que les simulations numériques ont été effectuées en utilisant le cône équivalent à l'indenteur Berkovich (angle d'attaque : $19,7^{\circ}$ ), ce qui permet de simplifier grandement les calculs.

Les résultats de densifications résiduelles alors obtenus sont en très bon accord avec les résultats expérimentaux (Fig. 5). Cette figure nous montre aussi que la taille de la zone densifiée est de l'ordre de la taille du contact dans le cas d'une indentation conique de type « Berkovich équivalent ». 

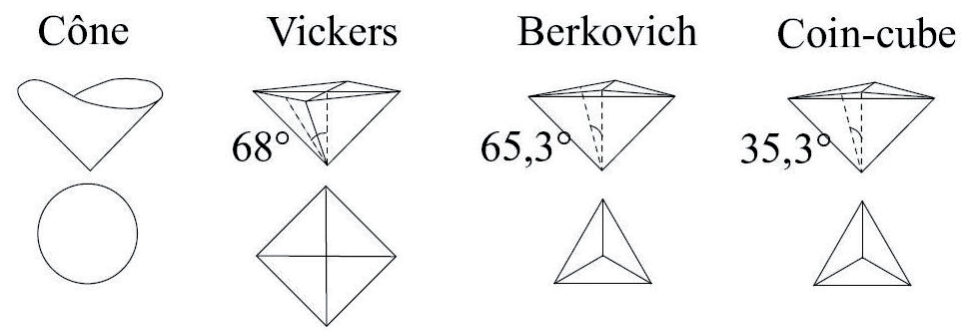

Fig. 3. Différentes géométries d'indenteurs utilisées couramment en indentation instrumentée.

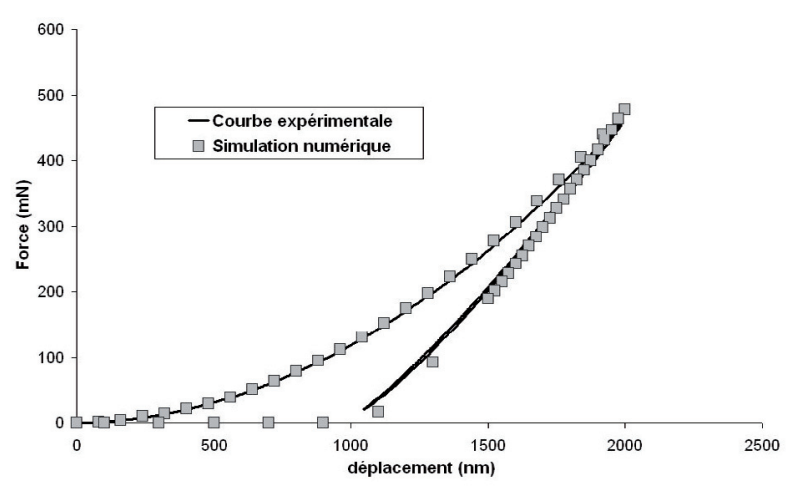

Fig. 4. Courbe charge-décharge de nano-indentation instrumentée avec une pointe Berkovich et une limite d'élasticité en cisaillement de $6500 \mathrm{MPa}$.

\subsection{Indentation Vickers tridimensionnelle}

Les cartographies de densification de Perriot [7] ont été mesurées après micro-indentation par une pointe Vickers tandis que les courbes de nano-indentation sont réalisées par indentation Berkovich. Dans les deux cas, les courbes charge-décharge sont quasiment confondues et peuvent être obtenues par une indentation conique équivalente d'angle d'attaque : $19,7^{\circ}$. Cependant, les géométries des pointes coniques, Vickers - pyramide à base carrée - et Berkovich - pyramide à base triangulaire - étant assez différentes (Fig. 3), les cartographies de densification peuvent être aussi relativement différentes, bien que la distribution caractéristique de densification soit sensiblement la même : forme de «bols empilés ». C'est pourquoi nous avons réalisé des simulations numériques d'indentations Vickers tridimensionnelles. Pour des raisons de symétrie, un huitième seulement de la géométrie est modélisé (Fig. 6). La discrétisation ainsi que les conditions aux limites utilisées sont similaires à celles proposées par Giannakopoulos et al. [24].

Les cartographies de densification mesurées expérimentalement par Perriot [7], Perriot et al. [14] en surface et celles obtenues par simulation numérique (Fig. 7) sont, là aussi, en très bon accord. Notons que les valeurs près de la pointe sont relativement différentes mais ceci provient essentiellement d'erreurs numériques liées à la singularité de la pointe. En revanche, la forme générale de la distribution de densification est bien reproduite. Comme dans le cas de l'indentation conique, remarquons que la taille de la zone densifiée est de l'ordre de grandeur de la taille du contact.

\subsection{Indentation « coin de cube »}

Nous avons vu ci-dessus que la loi de comportement développée dans ce document permet de bien reproduire les cartographies de densification mesurées à l'aide d'une micro-indentation Vickers. Les courbes de charge-décharge mesurées expérimentalement par nano-indentation Berkovich et obtenues par simulations numériques sont aussi en bon accord. Mais ceci est normal car c'est justement cette mesure qui nous a permis d'identifier la limite d'élasticité en cisaillement $q_{\mathrm{c}}$. Que se passe-t-il alors, si un indenteur plus «pointu» que la pointe Vickers (ou Berkovich) est utilisé? Ceci se traduit, du point de vue de l'indentation, par une déformation imposée au matériau bien plus importante [25, 26]. Par conséquent la loi de comportement développée dans ce document peut ne plus être vraiment adaptée.

Pour cette étude, nous avons choisi l'indenteur « coin de cube $\gg$ (Cube Corner sur la Fig. 3) qui peut être modélisé par un cône équivalent d'angle d'attaque $47,7^{\circ}$. Des essais de nano-indentation sur verres de silice ont aussi été réalisés. L'accord entre les courbes de chargedécharge mesurées expérimentalement et obtenues par la simulation numérique (Fig. 8) est assez satisfaisant. Remarquons que la courbe de charge obtenue par simulation numérique est en dessous de la courbe de charge mesurée expérimentalement. Ceci peut s'expliquer par le fait que le contact entre l'indenteur et la matière est supposé sans frottement dans la simulation numérique. Pour des indenteurs du type Berkovich ou Vickers, le frottement a peu d'effet car l'angle d'attaque est relativement faible (environ $20^{\circ}$ ). Dans le cas de l'indenteur « coin de cube $»$, ceci n'est plus vrai [27], ce qui explique une courbure plus importante de la courbe de charge mesurée expérimentalement.

Contrairement à l'indenteur Vickers, la microindentation « coin de cube » provoque une fissuration excessive du verre ce qui rend quasi-impossible la mesure de densification par micro-spectroscopie Raman. Lors d'une nano-indentation « coin de cube », la fissuration est bien moindre, mais il n'est pas possible de mesurer la densification à une aussi petite échelle avec cette technique. Nous n'avons donc pas accès à une cartographie de densification expérimentale avec cet indenteur. Nous avons 


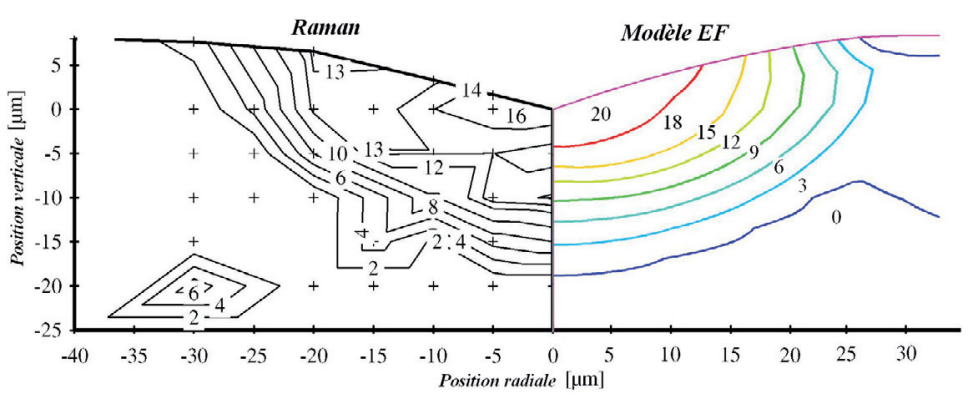

Fig. 5. Cartographie de densification $\left(\rho_{0}-\rho\right) / \rho_{0}$ sur une coupe après indentation. À gauche : mesure par micro-spectroscopie Raman [14], à droite : simulation numérique par éléments-finis [18].

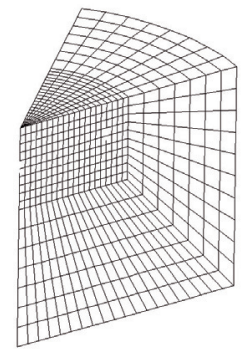

Fig. 6. Maillage tridimensionnel utilisé pour la simulation numérique de l'indentation Vickers. En raison des conditions de symétrie, seul un huitième du massif est modélisé.

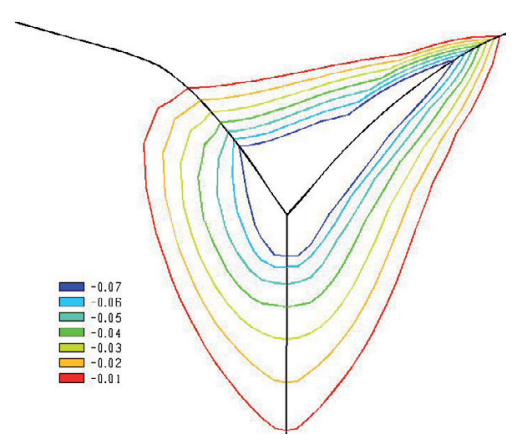

Fig. 7. Cartographie de déformation moyenne $(1 / 3 \operatorname{tr} \varepsilon)$ obtenue par simulation numérique par éléments-finis de l'indentation Vickers.

tout de même tracé sur la figure 9, la distribution de déformation moyenne (densification/3) obtenue par simulation numérique. La forme caractéristique dite de « bols empilés » est aussi observée, bien que l'indentation Cube Corner soit bien plus sévère que l'indentation Berkovich ou Vickers. Comme dans le cas de l'indentation Vickers, la taille de la zone densifiée est de l'ordre du rayon de contact entre l'indenteur et le massif. Ce résultat est particulièrement intéressant du fait qu'il semble possible de prédire a priori la taille de la zone densifiée sous contact par la mesure de l'aire de contact, et ceci quelle que soit l'apparente sévérité de l'indentation.

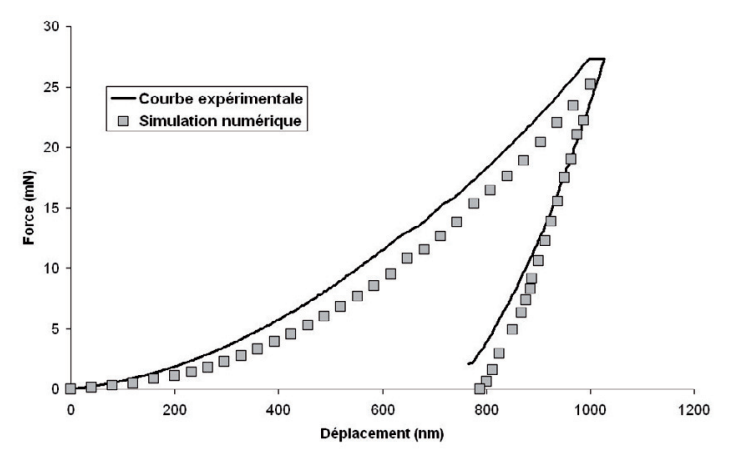

Fig. 8. Courbe charge-décharge de nano-indentation instrumentée avec une pointe «Coin de Cube» et une limite d'élasticité en cisaillement de $6500 \mathrm{MPa}$.

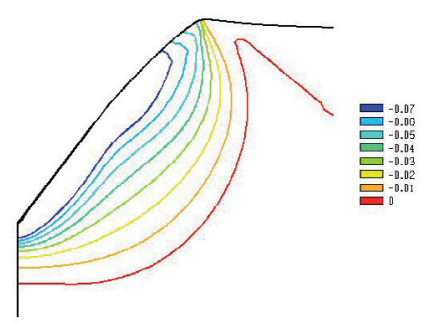

Fig. 9. Cartographie de déformation moyenne $(1 / 3 \operatorname{tr} \varepsilon)$ obtenue par simulation numérique éléments-finis. Vue en coupe après indentation « Coin de Cube ».

\section{3 À propos de l'essai d'indentation instrumentée}

La loi de comportement décrite dans cet article semble donc correctement décrire la densification des verres silicatés sous une sollicitation de type indentation instrumentée. Cependant les phénomènes d'écrouissage ou d'adoucissement sous des sollicitations déviatoriques pures ne peuvent être modélisés avec cette loi de comportement. Ceci montre que l'essai d'indentation instrumentée, bien que complété par des cartographies de densification, ne nous permet pas d'identifier totalement le comportement des verres silicatés à l'échelle micronique. D'autres types de tests doivent donc être utilisés pour améliorer la loi de comportement développée dans ce document. 


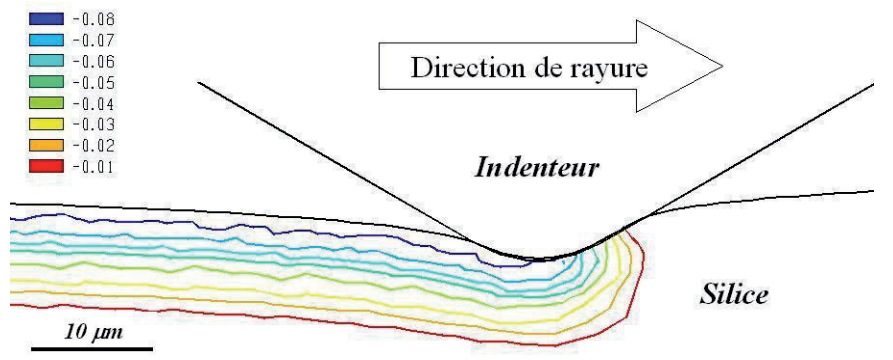

Fig. 10. Cartographie de déformation plastique moyenne $\left(1 / 3 \operatorname{tr} \varepsilon^{\mathrm{p}}\right)$ dans l'épaisseur du matériau après rayure par un dièdre arrondi. La zone densifiée est de l'ordre de grandeur de la longueur du contact.

\section{Simulation numérique de l'essai de rayure}

Un test plus complet que l'essai d'indentation en terme de déformations est l'essai de rayure. De plus, ce dernier nous intéresse plus particulièrement pour améliorer notre compréhension de la rayabilité des verres à l'échelle micronique. Malheureusement, l'analyse de cet essai est bien plus complexe que l'analyse de l'essai d'indentation [27]. Dans cette section, nous présentons des premiers résultats de densification calculés à partir d'une simulation numérique bidimensionnelle de rayure. Nous avons utilisé un indenteur rigide de type dièdre arrondi (angle d'attaque : $30^{\circ}$, rayon de pointe : $15 \mu \mathrm{m}$ ). Le contact entre l'indenteur et la silice est glissant (pas de frottement interfacial) et la profondeur de rayure est $15 \mu \mathrm{m}$. La loi de comportement décrite par les équations (1) et (2) a été utilisée pour modéliser le comportement des verres silicatés. Dans une première approche, seuls les résultats en phase stationnaire de rayure sont analysés. Cette phase stationnaire est obtenue lorsque la distribution de déformation plastique dans l'épaisseur du matériau après rayure devient indépendante de l'abscisse de mesure [27,28]. Il est alors possible d'observer une distribution caractéristique de l'état de densification du matériau (Fig. 10). Cette première simulation montre que la densification maximale a lieu en surface et qu'elle décroît de façon monotone dans l'épaisseur du matériau. Comme dans le cas de l'indentation, la taille de la zone densifiée est de l'ordre de grandeur de la longueur de contact entre le dièdre et le matériau.

\section{Conclusion}

Dans cet article, une loi de comportement élastoplastique permettant de modéliser la densification de la silice fondue à l'échelle micronique a été présentée. Les premiers résultats montrent le potentiel de cette loi pour améliorer notre compréhension de la plasticité des verres silicatés à l'échelle micronique sous des sollicitations de type indentation-rayure. D'un point de vue pratique, les résultats obtenus dans cette étude montre que la taille de la zone densifiée d'un verre de silice sous une action de contact est de l'ordre de grandeur de la taille du contact, et ceci quelle que soit la sévérité du contact (indentation-rayure). Ainsi, il semble possible de prédire a priori cette zone densifiée par la seule connaissance de la micro-géométrie du contact (rugosité, particules abrasives) et des efforts de contact.

Bien que les résultats semblent prometteurs, un problème important subsiste quant à l'écoulement plastique déviatorique qui n'est que très grossièrement pris en compte dans cette approche, faute de résultats expérimentaux adaptés. De nouveaux développements numériques et expérimentaux sont actuellement en cours en collaboration avec Saint-Gobain Recherche, notamment l'utilisation de nano-piliers.

\section{Références}

[1] J. Zarzycki, Glasses and the vitreous state, Cambridge University Press, 1991

[2] E. Barthel, Mécanique de surface du verre et physicochimie d'interface, Thèse HDR, 2006

[3] Y.W. Rhee, H.W. Kim, Y. Deng, B.R. Lawn, Brittle fracture versus quasi plasticity in ceramics: A simple predictive index, J. Am. Ceram. Soc. 84 (2001) 561-565

[4] E.W. Taylor, Plastic deformation of optical glasses, Nature 163 (1949) 323-325

[5] F.M. Ernsberger, Role of densification in deformation of glasses under point loading, J. Am. Ceram. Soc. 51 (1968) 545-547

[6] H. Sugiura, T. Yamadaya, Raman scattering in silica glass in the permanent densification region, J. Non-Cryst. Solids 144 (1992) 151-158

[7] A. Perriot, Nanoindentation de couches minces déposés sur substrat de verre de silice, Thèse de doctorat, Paris 6, 2005

[8] A.S. Argon, Plastic deformation in metallic glasses, Acta Metall. 27 (1979) 47-58

[9] M.L. Falk, J.S. Langer, Dynamics of viscoplastic deformation in amorphous solids, Phys. Rev. E 57 (1998) 71927205

[10] C.A. Schuh, T.C. Hufnagel, U. Ramamurty, Mechanical behavior of amorphous alloys, Acta Mater. 55 (2007) 4067-4109

[11] J.-L. Loubet, J.-M. Georges, O. Marchesini, G. Meille, Vickers indentation curves of magnesium oxydes, J. Tribology 106 (1984) 43-48

[12] W.-C. Oliver, G. Pharr, An improved technique for determining hardness and elastic modulus using load and displacement sensing indentation experiments, J. Mat. Res. 7 (1992) 1564-1583

[13] W.-C. Oliver, G. Pharr, Measurement of hardness and elastic modulus by instrumented indentation: Advances in understanding and refinements to methodology, J. Mat. Res. 19 (2004) 3-20

[14] A. Perriot, V. Martinez, L. Grosvalet, Ch. Martinet, B. Champagnon, D. Vandembroucq, E. Barthel, Raman microspectrocopic characterization of amorphous silica plastic behavior, J. Am. Ceram. Soc. 89 (2006) 596-601

[15] H. Sugiura, R. Ikeda, K. Kondo, T. Yamadaya, Densified silica after shock compression, J. App. Phys. 81 (1997) $1651-1655$ 
[16] F.L. Galeneer, Planar rings in glasses, Solid State Comm. 44 (1982) 1037-1040

[17] J.M. Besson, P.P. Pinceaux, Uniform stress conditions in the diamond anvil cell at 200 kilobars, Rev. Sci. Instr. 50 (1979) 541-543

[18] G. Kermouche, E. Barthel, D. Vandembroucq, P. Dubujet, Mechanical modelling of indentation-induced densification in amorphous silica, Acta Materialia, (2008), in press

[19] P.Y. Hicher, J.F. Shao, Modèle de comportement des sols et des roches, éd. Hermes science, 2002

[20] S. Brown, G. Abou-Chedid, Yield behavior of metal powder assemblages, J. Mech. Phys. Solids 42 (1994) 383-399

[21] S. Shima, M. Oyane, Plasticity theory for porous metals, Int. J. Mat. Sci. 18 (1978) 285-291

[22] Systus/Sysweld, User's manual. ESI Group, 2004

[23] T. Belytschko, W. Kam Liu, B. Moran, Non linear finite elements for continua and structures, Wiley, 2000
[24] A.E. Giannakopoulos, P.L. Larsson, R. Vestergaard, Analysis of Vickers indentation, Int. J. Solids Structures 31 (1994) 2679-2708

[25] G. Kermouche, J.-L. Loubet, J.-M. Bergheau, An approximate solution for the problem of cone or wedge indentation of elastoplastic solids, Comptes Rendus de Mécanique 333 (2005) 389-395

[26] G. Kermouche, J.-L. Loubet, J.-M. Bergheau, Extraction of stress-strain curves of elastic-viscoplastic solids using conical/pyramidal indentation testing with application to polymers, Mech. Mat., (2007), in press

[27] G. Kermouche, Contribution à la modélisation théorique et numérique des essais d'indentation et de rayure, Thèse de doctorat, École Centrale de Lyon, 2005

[28] G. Kermouche, A.-L. Kaiser, P. Gilles, J.-M. Bergheau, Combined numerical and experimental approach of the impact-sliding wear of a stainless steel in a nuclear reactor, Wear 263 (2007) 1551-1555 